\title{
Three-dimensional periodic motion in the vicinity of the equilibrium points of an asteroid
}

\begin{abstract}
O. Vasilkova
Pulkovo Observatory, St.-Petersburg, Russian Academy of Sciences; Isaac Newton Institute of Chile, St.-Petersburg Branch e-mail: olyaov@mail.ru

Received 29 September 2003 / Accepted 14 September 2004

Abstract. A way is described to find the initial conditions for the simplest three-dimensional periodic motions (3DPMs) in the vicinity of the equilibrium points (EPs) of an elongated asteroid whose shape is approximated by a triaxial ellipsoid (TE). A condition for the existence of these 3DPMs is formulated which may or may not be satisfied, depending on the spin period of the asteroid. A closed integral form of the gravitational potential of a TE is used. The general formulae for the computation of the initial conditions and some parameters of a 3DPM are derived from analysing the variational equations of motion. These initial conditions are used to integrate numerically the non-linear equations of motion in the vicinity of the EPs of a specific model of a triaxial ellipsoid based on the asteroid 243 Ida for a number of different rotation periods. We found that the period and some parameters of a 3DPM obtained by the numerical integration (with initial conditions predicted from the variational equations) have approximately the values computed from the variational equations.

We show that the equatorial projections of all 3DPMs constructed in the current work are ellipses with sizes dependent on one free parameter. General formulae are presented for the computation of the ratio of the semi-axes of the ellipse and the period of motion in it.

A special study is made for a boundary case where the EPs lying along the greatest ellipsoid axis (strongly unstable) are touching the asteroid surface. This critical value of the spin period of the asteroid (at which the loss of loose regoliths or destruction of the asteroid can start) is shown to be the same for the asteroids with similar densities and axial ratios.

Some comments are given on the condition under which a 3-D periodic motion can exist in the vicinity of the EPs of the Jacobi ellipsoid.
\end{abstract}

Key words. celestial mechanics - methods: analytical - methods: numerical - minor planets, asteroids

\section{Introduction}

There is a wealth of classical results pertaining to planar periodic orbits in the vicinity of the EPs (equilibrium points) and to the stability of the EPs of a near-spherical triaxial ellipsoid. The existence of the EPs in the vicinity of a nearspherical, triaxial, uniformly rotating ellipsoid was considered in Batrakov (1957) and their linear stability was studied by Abalakin (1957). The gravitational potential of a near-spherical triaxial ellipsoid was expressed there as a truncated harmonic expansion up to the second harmonic. In Batrakov (1957) the four equilibrium points were found to be situated on the extensions of the minor and major axes of the equatorial section of a TE (triaxial ellipsoid), called the "center" and the "saddle" equilibrium points (CEPs and SEPs) according to Scheeres' (1994) terminology. The coordinates of the equilibrium points were represented as a power series with respect to a small parameter. In Abalakin (1957) the linear stability of motion in the CEPs and SEPs was investigated and for a near-spherical TE the CEPs were proved to be linearly stable and the SEPs to be unstable. Two families of planar periodic orbits (of long and short periods) in the vicinity of the CEPs, and two families (short-periodic and asymptotic) in the vicinity of the SEPs were found to lie in the equatorial plane of the ellipsoid. The shape of all planar periodic orbits was shown to be elliptical. Further, the motion in the vicinity of the EPs of a rotating TE and stability of the EPs was investigated in many works. In Aksenov (1960) the gravitational potential of a body that is dynamically symmetrical with respect to its main planes of inertia is expressed as a truncated expansion, the coefficients of which are represented as linear combinations of the moments of inertia of a certain order. Aksenov investigates the linear stability of motion in the vicinity of the EPs for the case where the ratio of the spin rate squared to the mass of an ellipsoid is a small quantity. Nikolaev $(1968,1969,1972)$ has analysed the motion in the vicinity of the CEPs of a rotating near-spherical TE under the action of a perturbing body. The potential is expressed as a truncated harmonic expansion, again, up to the second harmonic. The spin rate of the ellipsoid is assumed to be low. In Zhuravlev $(1972,1973,1974)$ the stability of the CEPs for the planar and three-dimensional cases is studied using an expansion of the Hamiltonian up to the fourth and sixth order. The shape of the ellipsoid is close to spheroidal. In Kosenko (1981, 1981a) the geometrical properties of the area of stability of the CEPs are studied. The gravitational potential of a TE is given 
in a closed integral form. Conclusions about stability of the CEPs are made under the assumption that either the body has a near-spherical shape, with the libration points at any distance from the asteroid center, or it has an arbitrary ellipsoidal shape, with the libration points far from the asteroid. All the abovementioned works aimed at studying the motions in the vicinity of the libration points of a planet-like near-spherical body where effects of a deviation of the body's shape from spherical are relatively small compared to the attraction of the central body. It made it possible to use the gravitational harmonics formulation for representing the gravitational potential of a TE.

For orbits around a very elongated body (such as an asteroid) these classical analyses may no longer apply due to divergence of the series representing the potential within a sphere circumscribed around the body or due to very slow convergence of the series when close to this sphere (Subbotin 1949). Then it is convenient to concentrate on a closed form for the gravitational potential of a TE which would make it possible to study motion close to a TE of any elongation and spin rate. Antonov (1961) uses the closed integral form of the gravitational potential of an elongated triaxial ellipsoid for studying the formation of arms in SB galaxies. Two singular points of the surface situated at the extremities of the major axis of the ellipsoid are considered when the angular velocity reaches a certain limit and ejection of matter from these two points sets in. Danby (1965) uses the same closed form of the gravitational potential to investigate the motion in the vicinity of the SEPs of a triaxial ellipsoid when accounting for the formation of arms in barred spirals. (Different approaches should be mentioned for the study of the stability of motion in the vicinity of the equilibrium points of an elongated body which can be found in Contopoulos (1973), Lynden-Bell (1979), Colin (1979), Papayannopoulos (1979, 1979a), Schwarz (1984), Pfenniger (1990), etc., where orbits near corotation in spiral and barred galaxies are under consideration.)

Chauvineau et al. (1993) also use the closed form of the triaxial ellipsoid potential to investigate the motion close to an elongated triaxial ellipsoidal primary with axial ratios $\sqrt{2}: 1: 1 / \sqrt{2}$. A sizeable chaotic zone appears near $1 / 1$ resonance between the spin period of the primary and the period of revolution of its satellite in fast rotation. That is, the CEPs of the primary being stable at the primary's spin periods of 40, 20 and $10 \mathrm{~h}$, lose their stability at the faster rotation (with a period as short as $5 \mathrm{~h}$ in that paper) revealing a severe instability in near-synchronous orbits. The dependence of the linear stability of the EPs of an elongated TE on one parameter, which is a function of the axial ratios, density and spin rate of a homogeneous TE, has been studied in detail in Scheeres $(1993,1994)$ where the advantage of a classical closed form of the gravitational potential of a TE is used. No restrictions are imposed on the asteroid's elongation, density or spin rate. The conclusion is drawn that the linear stability of motion in the vicinity of the CEPs of an asteroid of a given triaxial ellipsoidal shape is violated by either decreasing the asteroid density or increasing its spin rate. Two classes of TEs are defined according to whether two of the sychronous orbits are stable or not (that is whether the CEPs of the ellipsoid are stable or not). In Vasilkova (1999) the dependence of both the linear stability of the CEPs and the positions of the SEPs and the CEPs on the spin rate of a rotating TE of a constant density for several specific models of ellipsoids (one of which, with semi-axes 350, 60, $10 \mathrm{~km}$, approaches a cigar-like shape) is under study. It is shown that for more elongated ellipsoids the CEPs lose their stability at a smaller spin rate. It turned out that the stable CEPs of an elongated TE can become unstable if a TE rotates fast, if its density is low or if its shape deviates significantly from spherical, i.e. if it is asteroid- or comet-like. This is contrary to traditional conclusions about the stability of the CEPs of a TE reached in studies using a truncated expansion up to a few (generally two) terms, allowing investigation only of the CEPs of TEs that have a near-spherical shape or of the CEPs which are far from the TE (at any its shape). In the current study a closed integral form of the gravitational potential of a homogeneous uniformly rotating TE is used to study the motion in the vicinity of the EPs of an asteroid (ellipsoid) of any elongation and spin rate. The aim of the present work is the analytical evaluation of the initial conditions for the simplest symmetrical (i.e. with at least one plane of symmetry) 3DPMs (three-dimensional periodic motions) in the vicinity of the EPs of a TE and the construction of these 3DPMs by numerical integration of the non-linear equations of motion, while the preceding works mentioned (except that of Scheeres 1993) concentrated on planar periodic motion in the vicinity of the EPs of a TE (i.e. the motion in the equatorial plane of an asteroid). This study also aims at pointing out the condition (in terms of the asteroid spin period) for which these 3DPMs exist in the vicinity of the EPs of a specific triaxial ellipsoid, assuming its shape and density fixed. This approach is different from that of Scheeres (1993) where a family of synchronous 3DPMs associated with a CEP of the Eros-based TE is computed for a particular value of the ellipsoid's spin period.

\section{Description of the problem}

Let us define a coordinate reference frame centered at an equilibrium point, so that new particle coordinates $\xi, \eta, \zeta$ are given by:

$$
\xi=x-x_{0}, \quad \eta=y-y_{0}, \quad \zeta=z-z_{0},
$$

where $x_{0}, y_{0}, z_{0}$ are the coordinates of the EP in a TE-centered coordinate frame with the $x, y, z$ axes aligned with the largest, intermediate and smallest principal axes of a TE, respectively. Then the variational equations of the particle motion in the vicinity of the EP are as follows (Subbotin 1937):

$$
\begin{aligned}
\ddot{\xi}-2 \omega \dot{\eta} & =\Omega_{x}+\xi \Omega_{x x}+\eta \Omega_{x y}+\zeta \Omega_{x z} \\
\ddot{\eta}+2 \omega \dot{\xi} & =\Omega_{y}+\xi \Omega_{y x}+\eta \Omega_{y y}+\zeta \Omega_{y z} \\
\ddot{\zeta} & =\Omega_{z}+\xi \Omega_{z x}+\eta \Omega_{z y}+\zeta \Omega_{z z}
\end{aligned}
$$

where $\xi, \eta, \zeta$ are the components of the displacement of a particle from the EP, $\omega$ is the spin rate of an asteroid,

$\Omega=\omega^{2}\left(x^{2}+y^{2}\right) / 2+V$

and the partial derivatives of $\Omega$ are taken at the EP under consideration. Given a constant density $\rho$ for a TE with semiaxes 
$a>b>c$, the classical closed form for the TE gravitational potential in a TE-fixed coordinate frame can be written as (Subbotin 1949):

$V=k^{2} \pi \rho a b c \int_{\lambda}^{+\infty}\left(1-\frac{x^{2}}{a^{2}+s}-\frac{y^{2}}{b^{2}+s}-\frac{z^{2}}{c^{2}+s}\right) \frac{\mathrm{d} s}{R(s)}$,

where $k$ is the Gauss constant,

$R(s)=\sqrt{\left(a^{2}+s\right)\left(b^{2}+s\right)\left(c^{2}+s\right)}$,

and $\lambda$ is the positive root of the cubic equation

$\frac{x^{2}}{a^{2}+\lambda}+\frac{y^{2}}{b^{2}+\lambda}+\frac{z^{2}}{c^{2}+\lambda}=1$.

As is known (Batrakov 1957), for a homogeneous triaxial ellipsoid there are four EPs lying on the continuation of its greatest and intermediate axes, i.e. the saddle equilibrium points (SEPs) and the center equilibrium points (CEPs), respectively. The SEP and CEP locations in a rotating frame may be computed by solving the following equations (Batrakov 1957):

$\Omega_{x}=\omega^{2} x+V_{x}=0$,

$\Omega_{y}=\omega^{2} y+V_{y}=0$,

or the equations (Antonov 1961; Danby 1965; Scheers 1993; 1994; Vasilkova 1999)

$\omega^{2}-\frac{3}{2} k^{2} M \int_{\lambda}^{+\infty} \frac{\mathrm{d} s}{\left(a^{2}+s\right) R(s)}=0$,

$\omega^{2}-\frac{3}{2} k^{2} M \int_{\lambda}^{+\infty} \frac{\mathrm{d} s}{\left(b^{2}+s\right) R(s)}=0$,

where $M$ is the mass of the asteroid. (See Scheeres 1994, for a discussion of the conditions for the existence of the EPs.) Computations show that the distance of the EPs from the center of the ellipsoid decreases with increasing ellipsoid spin rate. On the other side, for different ellipsoids rotating with the same spin rate the distance mentioned is shorter for a more elongated ellipsoid, that is, for one that has higher ratios $a / c$ and $b / c$ of its semiaxes $a>b>c$ (Vasilkova 1999). Due to the symmetry of the positions of the equilibrium points, with coordinates $\left(x_{0}, 0,0\right)$ and $\left(-x_{0}, 0,0\right),\left(0, y_{0}, 0\right)$ and $\left(0,-y_{0}, 0\right)$ with respect to the center of inertia of the ellipsoid, it is sufficient to study only the points $\left(x_{0}, 0,0\right)$ and $\left(0, y_{0}, 0\right)$, where $x_{0}>0, y_{0}>0$. Evaluating the first and second partial derivatives of $\Omega$ at these EPs yields:

$\Omega_{x}=\Omega_{y}=\Omega_{z}=\Omega_{x y}=\Omega_{y x}=\Omega_{x z}=\Omega_{z x}=\Omega_{y z}=\Omega_{z y}=0$,

so Eqs. (2)-(4) reduce to

$\ddot{\xi}-2 \omega \dot{\eta}=\xi \Omega_{x x}$,

$\ddot{\eta}+2 \omega \dot{\xi}=\eta \Omega_{y y}$,

$\ddot{\zeta}=\zeta \Omega_{z z}$.
It is seen that the solution of Eqs. (14)-(15) is independent of the solution of Eq. (16). So the periodic solution of Eqs. (14)-(15) represents just the $\xi \eta$ (or $x y$ ) component of a potential 3-D periodic solution of Eqs. (14)-(16). Searching for the solutions of Eqs. (14)-(15) in the form

$\xi=P \mathrm{e}^{\sigma t}, \quad \eta=Q \mathrm{e}^{\sigma t}$,

where $P, Q$ are constants, leads to the characteristic equation

$\widetilde{\sigma}^{4}+\widetilde{\sigma}^{2}\left(4-\widetilde{\Omega}_{x x}-\widetilde{\Omega}_{y y}\right)+\widetilde{\Omega}_{x x} \widetilde{\Omega}_{y y}=0$,

where

$\widetilde{\sigma}=\sigma / \omega, \quad \widetilde{\Omega}_{x x}=\Omega_{x x} / \omega^{2}, \quad \widetilde{\Omega}_{y y}=\Omega_{y y} / \omega^{2}$.

The motion in the vicinity of an EP lying in the equatorial plane (that is, the $\xi \eta(x y)$ projection of a 3-D motion) is stable only when this quadratic (with respect to $\widetilde{\sigma}^{2}$ ) characteristic equation has two real unequal negative roots (Lyapunov 1950), that is, by the well-known properties of the roots of a quadratic equation the following inequalities must hold:

$C=\widetilde{\Omega}_{x x} \widetilde{\Omega}_{y y}>0$,

$B=-\widetilde{\Omega}_{x x}-\widetilde{\Omega}_{y y}+4>0$,

$D=\left(-\widetilde{\Omega}_{x x}-\widetilde{\Omega}_{y y}+4\right)^{2}-4 \widetilde{\Omega}_{x x} \widetilde{\Omega}_{y y}>0$,

where the second partial derivatives $\widetilde{\Omega}_{x x}=1+V_{x x} / \omega^{2}$, $\widetilde{\Omega}_{y y}=1+V_{y y} / \omega^{2}$, are taken at the EP. (A thorough analysis of the conditions (20)-(22) with respect to the problem of linear stability of the EPs of a triaxial ellipsoid may be found in Scheeres 1994.)

At the SEP with coordinates $\left(x_{0}, 0,0\right)$ the expressions for the second partial derivatives can be reduced to

$\widetilde{\Omega}_{x x}=\frac{3 k^{2} M}{\omega^{2} x_{0} \sqrt{\left(b^{2}+\lambda\right)\left(c^{2}+\lambda\right)}}>0$,

$\widetilde{\Omega}_{y y}=1-\frac{3 k^{2} M}{2 \omega^{2}} \int_{\lambda}^{+\infty} \frac{\mathrm{d} s}{\left(b^{2}+s\right) R(s)}<0$,

where the inequality $\widetilde{\Omega}_{y y}<0$ is valid due to equality (11) at a SEP. That is, taking Eq. (11) into account, Eq. (24) reduces to

$\widetilde{\Omega}_{y y}=1-\int_{\lambda}^{+\infty} \frac{\mathrm{d} s}{\left(b^{2}+s\right) R(s)} / \int_{\lambda}^{+\infty} \frac{\mathrm{d} s}{\left(a^{2}+s\right) R(s)}<0$,

which is obvious as $b<a$. In the same way, at the CEP $\left(0, y_{0}, 0\right)$ we have

$\widetilde{\Omega}_{y y}=\frac{3 k^{2} M}{\omega^{2} y_{0} \sqrt{\left(a^{2}+\lambda\right)\left(c^{2}+\lambda\right)}}>0$,

$\widetilde{\Omega}_{x x}=1-\frac{3 k^{2} M}{2 \omega^{2}} \int_{\lambda}^{+\infty} \frac{\mathrm{d} s}{\left(a^{2}+s\right) R(s)}>0$, 
where inequality (27), in turn, is valid because identity (12) holds at a CEP. That is, taking Eq. (12) into account, Eq. (27) reduces to the obvious (as $a>b$ ) inequality

$\widetilde{\Omega}_{x x}=1-\int_{\lambda}^{+\infty} \frac{\mathrm{d} s}{\left(a^{2}+s\right) R(s)} / \int_{\lambda}^{+\infty} \frac{\mathrm{d} s}{\left(b^{2}+s\right) R(s)}>0$.

It is seen that condition (20) is violated at the SEP $\left(x_{0}, 0,0\right)$ at all values of $\omega, \rho$ and semiaxes $a>b>c$ of a triaxial ellipsoid. Therefore, the motion in the vicinity of a SEP will be unstable for any shape, density and rotation rate of a TE. At the CEP $\left(0, y_{0}, 0\right)$ condition (20) holds for all admissible $\omega, \rho$ and $a>b>c$ of a TE. So, the linear stability of motion at the CEP depends on the value of the left-hand side of inequalities (21) and (22) which can be either positive or negative depending on the shape, density and rotation rate of a TE. The more the ellipsoid is elongated the smaller the rotation rate at which inequalities (21) and (22) are violated (Vasilkova 1999).

We have considered the conditions for the periodic motion which is the $\xi \eta$ projection of a 3-D motion to be stable (in the linear sense) by analysing Eqs. (14)-(15) and derived the formulae for the quantities $B, C, D, \widetilde{\Omega}_{x x}, \widetilde{\Omega}_{y y}, \widetilde{\sigma}^{2}$ which are used for the computation of some parameters of a 3-D periodic motion (its period and ratio $q$ of the semiaxes of the $\xi \eta$ projection ellipse. Analogously, the out-of-plane linear stability can be established by considering Eq. (16) (see Eq. (44)). The corresponding characteristic equation has a pair of purely imaginary roots, so the corresponding out-of-plane periodic motion is conditionally stable in the linear sense.

\section{The shape of the $\xi \eta$ component of a 3-D periodic solution}

A periodic solution of Eqs. (14) and (15) for the motion of a particle in the vicinity of any EP of a triaxial ellipsoid searched for in the form $\xi=P \mathrm{e}^{\sigma t}, \eta=Q \mathrm{e}^{\sigma t}$ can be expressed as

$\xi=P_{1} \mathrm{e}^{\omega \sqrt{\widetilde{\sigma}^{2}} t}+P_{2} \mathrm{e}^{-\omega \sqrt{\widetilde{\sigma}^{2}} t}$,

$\eta=R_{1} \mathrm{e}^{\omega \sqrt{\widetilde{\sigma}^{2}} t}+R_{2} \mathrm{e}^{-\omega \sqrt{\sigma^{2}} t}$

where $\widetilde{\sigma}^{2}$ is a negative root of the quadratic (with respect to $\widetilde{\sigma}^{2}$ ) Eq. (18). These expressions can be reduced to the form:

$\xi=A_{1} \cos \left(\omega \sqrt{-\widetilde{\sigma}^{2}} t\right)+A_{2} \sin \left(\omega \sqrt{-\widetilde{\sigma}^{2}} t\right)$

$\eta=B_{1} \cos \left(\omega \sqrt{-\widetilde{\sigma}^{2}} t\right)+B_{2} \sin \left(\omega \sqrt{-\widetilde{\sigma}^{2}} t\right)$

where

$A_{1}=P_{1}+P_{2}, A_{2}=i\left(P_{1}-P_{2}\right)$,

$B_{1}=R_{1}+R_{2}, \quad B_{2}=i\left(R_{1}-R_{2}\right)$.

Using relationships between the coefficients $P_{1}, P_{2}$ and $R_{1}, R_{2}$ (which can be obtained by substituting the solution $\xi=P \mathrm{e}^{\sigma t}$,
$\eta=\mathrm{Re}^{\sigma t}$ into Eqs. (14), (15)), they reduce further to the equation of an ellipse:

$\frac{\xi^{2}}{q^{2}\left(B_{1}^{2}+B_{2}^{2}\right)}+\frac{\eta^{2}}{B_{1}^{2}+B_{2}^{2}}=1$.

The period of movement in the ellipse is equal to

$P=\frac{2 \pi}{\omega \sqrt{-\widetilde{\sigma}^{2}}}=\frac{T}{\sqrt{-\widetilde{\sigma}^{2}}}$,

where $T$ is the spin period of the asteroid, and $\widetilde{\sigma}^{2}$ is the negative root of the quadratic (with respect to $\widetilde{\sigma}^{2}$ ) Eq. (18). It is seen that the size of the ellipse depends on one free parameter equal to $B_{1}{ }^{2}+B_{2}{ }^{2}$. The ratio of the semiaxes of the ellipse is determined by the quantity $q$ which can be computed from the equation

$q=\frac{-\widetilde{\sigma}^{2}+\widetilde{\Omega}_{y y}}{2 \sqrt{-\widetilde{\sigma}^{2}}}$

So, if we search for a solution of the variational Eqs. (14), (15) in the form $\xi=P \mathrm{e}^{\sigma t}, \eta=R \mathrm{e}^{\sigma t}$, the projection of the corresponding 3-D periodic motion in the vicinity of any EP of a triaxial ellipsoid on its equatorial plane is an ellipse. (For a nearspherical TE this was proved in Abalakin (1957).) The roots $\widetilde{\sigma}^{2}$ are the functions of the quantities $B$ and $C$ (see Eqs. (77), (78)) which are functions of the second derivatives $\widetilde{\Omega}_{x x}, \widetilde{\Omega}_{y y}$. These derivatives can be computed using the formulae (23), (24) and (26), (27) for the SEPs and CEPs, correspondingly, and they in turn depend (as can be derived from the Eqs. (23), (24), (26), (27)) on the quantities $T^{2} \rho ; a / c ; b / c$ only, where $T$ is the spin period of the ellipsoid, $\rho$ is its density, and $a / c, b / c$ are the ratios of the ellipsoid semiaxes $a>b>c$. So the ratio $q$ of the semiaxes of the ellipse and the period $P$ of the movement in the ellipse depend on the parameters $T^{2} \rho ; a / c ; b / c$ of the ellipsoid only.

A general form of the solution of the variational equations of motion (14) and (15) in the vicinity of an EP is the following:

$\xi=P_{1} \mathrm{e}^{\omega \sqrt{\sigma_{1}^{2}} t}+P_{2} \mathrm{e}^{-\omega \sqrt{\sigma_{1}^{2}} t}+P_{3} \mathrm{e}^{\omega \sqrt{\sigma_{2}^{2}} t}+P_{4} \mathrm{e}^{-\omega \sqrt{\widetilde{\sigma}_{2}^{2}} t}$,

$\eta=R_{1} \mathrm{e}^{\omega \sqrt{\sigma_{1}^{2}} t}+R_{2} \mathrm{e}^{-\omega \sqrt{\sigma_{1}^{2}} t}+R_{3} \mathrm{e}^{\omega \sqrt{\sigma_{2}^{2}} t}+R_{4} \mathrm{e}^{-\omega \sqrt{\sigma_{2}^{2}} t}$,

where $\widetilde{\sigma}_{1}^{2}, \widetilde{\sigma}_{2}^{2}$ are the roots of the quadratic Eq. (18). It is seen that if at least one of the roots $\widetilde{\sigma}_{1}^{2}, \widetilde{\sigma}_{2}^{2}$ is negative, then for a certain choice of initial conditions one family of periodic motion exists, because we have a pair of purely imaginary roots (Lyapunov 1950).

\section{Three-dimensional periodic motions in the vicinity of the EPs}

Equation (16) has a solution independent of the solution of Eqs. (14) and (15). The expression for the second partial derivative $\Omega_{z z}$ taken at the EP yields

$\Omega_{z z}=-4 \pi k^{2} \rho \frac{a}{c} \frac{b}{c} \int_{0}^{u} \frac{t^{2} \mathrm{~d} t}{\left(1+l^{2} t^{2}\right)^{1 / 2}\left(1+l_{1}^{2} t^{2}\right)^{1 / 2}}<0$, 
where the values of the parameter

$u=c / \sqrt{c^{2}+\lambda}$

are equal to

$u_{\mathrm{SEP}}=1 / \sqrt{\frac{x_{0}^{2}}{c^{2}}-l^{2}}, \quad u_{\mathrm{CEP}}=1 / \sqrt{\frac{y_{0}^{2}}{c^{2}}-l_{1}^{2}}$

at the $\operatorname{SEP}\left(x_{0}, 0,0\right)$ and $\operatorname{CEP}\left(0, y_{0}, 0\right)$, respectively, $\lambda$ and $a>b>c$ are defined as before, and

$l=\sqrt{a^{2}-c^{2}} / c, \quad l_{1}=\sqrt{b^{2}-c^{2}} / c$

are so called secondary eccentricities. Because $\Omega_{z z}$ is always negative, the corresponding characteristic equation has a pair of purely imaginary roots and, thus, the solution of Eq. (16) is periodic (Lyapunov 1950). Its general form is:

$\zeta=C_{1} \mathrm{e}^{i \sqrt{-\Omega_{z z}} t}+C_{2} \mathrm{e}^{-i \sqrt{-\Omega_{z z}} t}$,

or,

$\zeta=C_{3} \cos \sqrt{-\Omega_{z z}} t+C_{4} \sin \sqrt{-\Omega_{z z}} t$,

where $C_{1}, C_{2}, C_{3}$ and $C_{4}$ are constant.

For a non-planar orbit to be periodic it is necessary that the period of oscillation $P_{\xi \eta}$ of the particle coordinates $\xi, \eta$ in an EP - centered coordinate frame would be equal to the period $P_{\zeta}$ of the oscillation of the coordinate $\zeta$ or, in general, the following equalities should hold:

$P_{\xi \eta}=\frac{m}{n} P_{\zeta}$,

$P_{\xi \eta}=\frac{T}{\sqrt{-\widetilde{\sigma}^{2}}}$,

$P_{\zeta}=\frac{2 \pi}{\sqrt{-\Omega_{z z}}}=\sqrt{\frac{\pi}{k^{2} \rho \frac{a}{c} \frac{b}{c} I_{3}}}$,

where

$I_{3}=\int_{0}^{u} \frac{t^{2} \mathrm{~d} t}{\left(1+l^{2} t^{2}\right)^{1 / 2}\left(1+l_{1}^{2} t^{2}\right)^{1 / 2}}$,

$m, n$ form an irreducible fraction. From Eqs. (11) and (12) the following expressions can be derived for the ellipsoid spin period in terms of the distance $x_{0}$ of the SEP or $y_{0}$ of the CEP from the ellipsoid center

$T=\sqrt{\frac{\pi}{k^{2} \rho \frac{a}{c} \frac{b}{c} I_{\mathrm{SEP}}}}, \quad T=\sqrt{\frac{\pi}{k^{2} \rho \frac{a}{c} \frac{b}{c} I_{\mathrm{CEP}}}}$,

correspondingly, where

$I_{\mathrm{SEP}}=\int_{0}^{u_{\mathrm{SEP}}} \frac{t^{2} \mathrm{~d} t}{\left(1+l^{2} t^{2}\right)^{3 / 2}\left(1+l_{1}^{2} t^{2}\right)^{1 / 2}}$,
$I_{\mathrm{CEP}}=\int_{0}^{u_{\mathrm{CEP}}} \frac{t^{2} \mathrm{~d} t}{\left(1+l^{2} t^{2}\right)^{1 / 2}\left(1+l_{1}^{2} t^{2}\right)^{3 / 2}}$,
Table 1. Some parameters of the 3-D periodic motions plotted below.

\begin{tabular}{ccccccc}
\hline \hline $\begin{array}{c}T \\
(\mathrm{~h})\end{array}$ & $\frac{P_{\xi \eta}}{P_{\zeta}}$ & $\begin{array}{r}P_{\xi \eta} \\
(\mathrm{h})\end{array}$ & $\begin{array}{r}P_{\zeta} \\
(\mathrm{h})\end{array}$ & $\begin{array}{r}P_{3 D} \\
(\mathrm{~h})\end{array}$ & $\begin{array}{c}\Delta \\
(\mathrm{cm})\end{array}$ & $\Delta q$ \\
\hline 18.368 & $3 / 2$ & 27.484 & 18.323 & 54.969 & 8.3 & 0.0006 \\
22.955 & $2 / 1$ & 45.827 & 22.914 & 45.827 & 0.1 & 0.0006 \\
29.977 & $5 / 2$ & 74.848 & 29.939 & 149.697 & 0.2 & 0.0005 \\
38.212 & $3 / 1$ & 114.532 & 38.177 & 114.532 & 1.6 & 0.0023 \\
47.355 & $7 / 2$ & 165.631 & 47.323 & 331.262 & 1.6 & 0.0018 \\
\hline
\end{tabular}

and $u_{\text {SEP }}$ and $u_{\text {CEP }}$ are defined by Eq. (42). Combining Eqs. (50) with Eq. (48) and remembering the generalized theorem about the mean value yields at the CEP

$\frac{c}{b} \leq \frac{P_{\zeta}}{T}<1$

and at the SEP

$\frac{c}{a} \leq \frac{P_{\zeta}}{T}<1$

Equation (46) states that the period of the 3DPM is defined by $P=m P_{\zeta}=n P_{\xi \eta}$. So, if $P_{\zeta}$ is commensurable with $T$ then $P$ is also commensurable with $T$, and inequalities (53) and (54) reduce at the CEP to

$m \frac{c}{b} \leq \frac{P}{T}<m$

and at the SEP to

$m \frac{c}{a} \leq \frac{P}{T}<m$

Particularly, it can be noticed from Eqs. (53), (55) (for the CEPassociated 3DPM) that for the asteroids having or approaching a two-axial ellipsoidal shape $(b=c)$ the period of the vertical oscillation $P_{\zeta}$ is equal or nearly equal to the asteroid spin period $T$, and the period $P$ of the possible 3DPM is approximately equal to $m T$. That is, for two-axial asteroids (ellipsoids) the period of the 3DPM is always commensurable with the asteroid spin period. For example, in Table 1 for 3-D periodic motions computed for the ellipsoid based on the asteroid Ida $(a=28, b=12, c=10.5 \mathrm{~km}, c / b=0.875)$ the period $P_{\zeta}$ is very close to the ellipsoid spin period within the chosen range of $T$ and the period $P$ is 3,2,5,3,7 times the ellipsoid spin period $T$ falling within the limits of inequality (55). In the same way, Eqs. (54), (56) show, specifically, that almostcommensurability of the 3-D motion with the ellipsoid spin period in the vicinity of the SEP would exist if the asteroid were possessed a near-spherical shape.

In Fig. 1 the 3-D periodic motion in the vicinity of the SEP of an Ida-like asteroid $(c / a=0.375)$ is represented when the period $P_{\zeta}$ is equal to the period $P_{\xi \eta}(m=1$ in Eq. (56)) and 


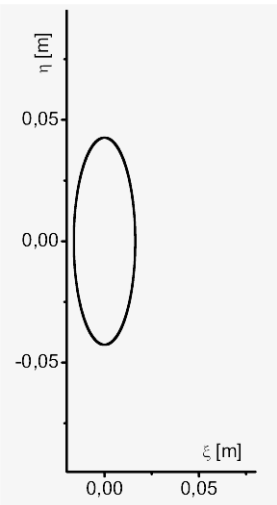

a)

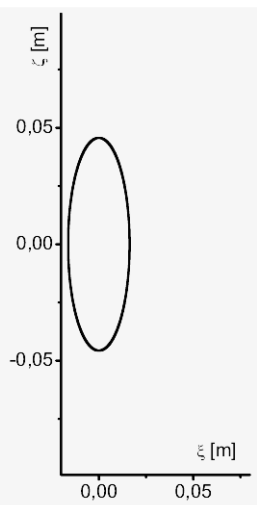

b)

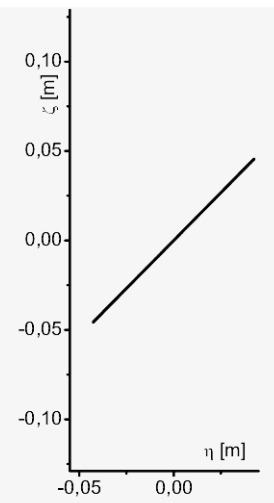

c)

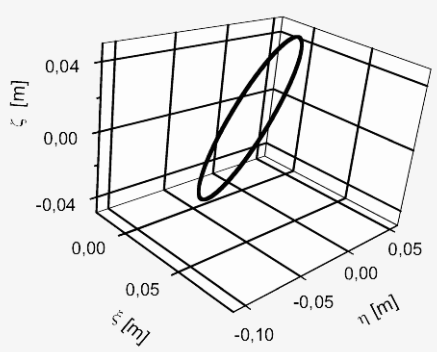

d)

Fig. 1. An unstable 3-D periodic motion of period $P=2.485 \mathrm{~h}$ in the vicinity of the SEP of an Ida-like triaxial ellipsoid at a spin period of the ellipsoid of $T=3.919 \mathrm{~h}$ and commensurability $P_{\zeta} / P_{\xi \eta}=1$ in the $\mathrm{SEP}$ - centered frame.

equal to $2.485 \mathrm{~h}$ (this is also the period $P$ of the $3-\mathrm{D}$ motion) while the ellipsoid spin period equals $3.919 \mathrm{~h}$. These values are in agreement with inequality (56) which yields $0.375 \leq$ $0.634<1$.

\section{Computation of initial conditions}

For the computations performed in the current study a specific model of a TE is chosen based on the asteroid Ida with semiaxes $a=28, b=12, c=10.5 \mathrm{~km}$ and density $2.5 \mathrm{~g} \mathrm{~cm}^{-3}$. First, a particular value of the spin period of the TE at which the variational Eqs. (14)-(16) admit a 3-D periodic solution of the described type is computed (see Eq. (46) and Figs. 6, 7). Writing out a general form of the 3-D periodic solution of Eqs. (14)-(16) (see Eqs. (29)-(32), (44), (45)) we get

$$
\begin{aligned}
& \xi=A_{1} \cos \left(\omega \sqrt{-\widetilde{\sigma}^{2}} t\right)+A_{2} \sin \left(\omega \sqrt{-\widetilde{\sigma}^{2}} t\right), \\
& \eta=B_{1} \cos \left(\omega \sqrt{-\widetilde{\sigma}^{2}} t\right)+B_{2} \sin \left(\omega \sqrt{-\widetilde{\sigma}^{2}} t\right), \\
& \zeta=C_{1} \cos \sqrt{-\Omega_{z z}} t+C_{2} \sin \sqrt{-\Omega_{z z}} t,
\end{aligned}
$$

where $A_{1}, A_{2}, B_{1}, B_{2}, C_{1}, C_{2}$ are constant. Differentiating the equations above we find the corresponding values for the initial coordinates, velocities and accelerations at a particular initial moment of time (in this paper we assume this to be zero).
Substituting the results into Eqs. (14)-(16) yields the following expressions for the initial conditions:

$\xi_{0}=A_{1}$,

$\eta_{0}=B_{1}$

$\dot{\xi}_{0}=\frac{-\widetilde{\sigma}^{2}+\widetilde{\Omega}_{y y}}{2} \omega \eta_{0}$,

$\dot{\eta}_{0}=-\frac{-\widetilde{\sigma}^{2}+\widetilde{\Omega}_{x x}}{2} \omega \xi_{0}$,

$\zeta_{0}=C_{1}$

$\dot{\zeta}_{0}=C_{2} \sqrt{-\Omega_{z z}}$,

where $\widetilde{\sigma}^{2}$ is a negative root of the quadratic (with respect to it) Eq. (18) and the second partial derivatives $\widetilde{\Omega}_{x x}, \widetilde{\Omega}_{y y}, \Omega_{z z}$ are taken at the point of equilibrium. There are four free parameters. Setting $A_{1}=C_{2}=0$, the constants $B_{1}, C_{1}$ can be obtained by the method of successive approximations so that the resulting 3-D periodic solution would have periods satisfying Eqs. (46)-(48). Particularly, for the 3DPMs demonstrated in Figs. 8-12 for the motion in the vicinity of the CEP $\left(0, y_{0}, 0\right)$, the constants above should be such that the resulting 3-D solutions would satisfy Eqs. (48), (85), (86). The root $\widetilde{\sigma}^{2}$ is computed using Eq. (78) corresponding to the longer period $P_{\xi \eta}=P_{2}$. In Table 1 the following periods of the constructed 3-D periodic motions are listed: $P_{\xi \eta}$ is the period of motion in the $\xi \eta$ projection of the 3 -D periodic motion, $P_{\zeta}$ is the period of oscillation in the $\zeta$ direction, $P_{3 D}$ is the period of the entire 3-D periodic motion, and $T$ is the spin period of the ellipsoid. The computed quantity $\Delta$ is the deviation of a particle from its initial position after a time interval which consists of an integer number of periods in the 3-D periodic orbit and which is nearly equal to 3 months. It is seen that the greatest deviation does not exceed $8.3 \mathrm{~cm}$ thus, we assume these 3DPMs to be stable.

\section{Periodic motions in the vicinity of the SEPs}

Equations (23), (24) show that at the SEP of any TE the product of two second partial derivatives of $\Omega$, that is the value of $C$ in Eq. (20), is negative. This means that one root $\widetilde{\sigma}^{2}$ of the quadratic Eq. (18) is negative, and we have one family of planar periodic motions in the vicinity of the SEP that is a family of possible 3-D periodic motions (provided that condition (46) holds) whose $\xi \eta$ projections are ellipses (Eq. (35)). Because the SEPs are unstable for any shape, density and rotation rate of a triaxial ellipsoid, the motion in the $\xi \eta$ projection and, consequently, the corresponding three-dimensional periodic motion is also unstable. Figure 1 demonstrates $\xi \eta, \xi \zeta$ and $\eta \zeta$ projections of an unstable 3DPM in the vicinity of the SEP of an Idalike TE. These are computed by numerical integration of the non-linear equations of motion in the $\operatorname{SEP}\left(x_{0}, 0,0\right)$ - fixed coordinate frame with the initial conditions obtained by solving the variational equations of motion (14)-(16). The ellipsoid spin period was taken to be equal to $T=3.919 \mathrm{~h}$ because a $3 \mathrm{DPM}$ is possible in this case due to the fact that Eq. (46) 
holds for $m / n=1$, that is, the period of movement in the ellipse lying in the equatorial plane of the ellipsoidal asteroid is equal to the period of the out-of-plane oscillation. As the real spin period of $243 \mathrm{Ida}$ is $4.63 \mathrm{~h}$, at this period a 3DPM searched for in the way described here is not possible in the vicinity of Ida's SEPs.

Note that the period of rotation at which the SEPs are touching the asteroid surface is the same for asteroids having the same density and ratios $a / c, b / c$ of their semiaxes $a>b>c$. Indeed, in general, the squared value of the period of rotation of a TE can be reduced to the expression:

$T^{2}=\frac{\pi}{k^{2} \rho \frac{a}{c} \frac{b}{c} I_{1}}$,

where

$I_{1}=\int_{0}^{u} \frac{t^{2} \mathrm{~d} t}{\left(1+l^{2} t^{2}\right)^{3 / 2}\left(1+l_{1}^{2} t^{2}\right)^{1 / 2}}$,

$u=1 / \sqrt{\frac{x_{0}^{2}}{c^{2}}-l^{2}}, \quad l^{2}=\frac{a^{2}}{c^{2}}-1, \quad l_{1}^{2}=\frac{b^{2}}{c^{2}}-1$,

$x_{0}$ is the distance of the SEP along the semiaxis $a$ from the asteroid center. Formula (66) shows that if for a certain value of spin period $T_{x_{0}=a}$ the saddle point of libration $\left(x_{0}, 0,0\right)$ approaches the surface of the ellipsoid (at $x_{0}=a$ ), the upper limit $u$ of the integral $I_{1}$ in Eqs. (66), (67) attains 1, and the integral gets a value which is the same for the asteroids with the same ratios $a / c, b / c$ of their semiaxes. Thus the value of $T^{2}$ is dependent only on the density and ratios of the semiaxes of a TE. So, as the saddle equilibrium points are highly unstable, the destruction at the longest ends of the asteroids can start at the same value of the spin period for asteroids of similar density and axial ratios (provided that there are no internal cohesive forces in addition to gravity). This critical value of the spin period is independent of the dimensions of an asteroid or of its mass. Its square is equal to

$T_{x_{0}=a}^{2}=\frac{\pi}{k^{2} \rho \frac{a}{c} \frac{b}{c} I_{0}}$,

where

$I_{0}=\int_{0}^{1} \frac{t^{2} \mathrm{~d} t}{\left(1+l^{2} t^{2}\right)^{3 / 2}\left(1+l_{1}^{2} t^{2}\right)^{1 / 2}}$.

For example, for the asteroid Ida the critical rotation period is $3.278 \mathrm{~h}$ (at its real spin period of $4.63 \mathrm{~h}$ ). If Ida had been rotating faster in the past, with a spin period of $3.278 \mathrm{~h}$ or shorter, the loose particles (or just some components of the rubble-pile which could represent Ida) at its longest ends would leave the surface and fly off. Perhaps it could help to explain the formation of the hollow observed at one of the ends of Ida and the existence of Ida's satellite Dactyl.

Note that if a triaxial ellipsoid is a Jacobi one, then the results above can be applied to its equilibrium points too, though the SEPs can never lie on the surface of the Jacobi ellipsoid (it is just the consequence of the Jacobi ellipsoid being a figure of equilibrium). The question of whether the approximation of

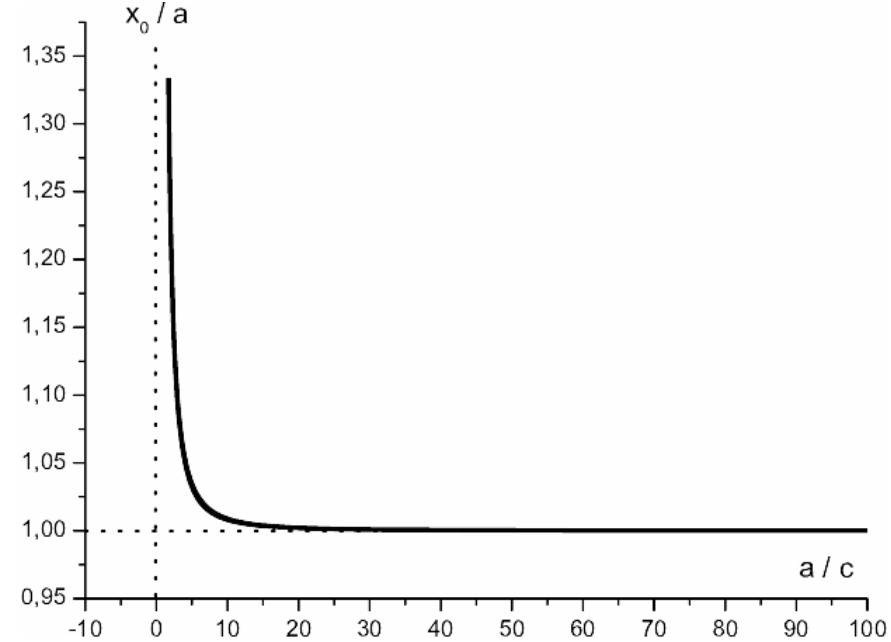

Fig. 2. The relative distance $x_{0} / a$ of the SEPs of a Jacobi ellipsoid from the center versus the ratio $a / c$ of the semiaxes.

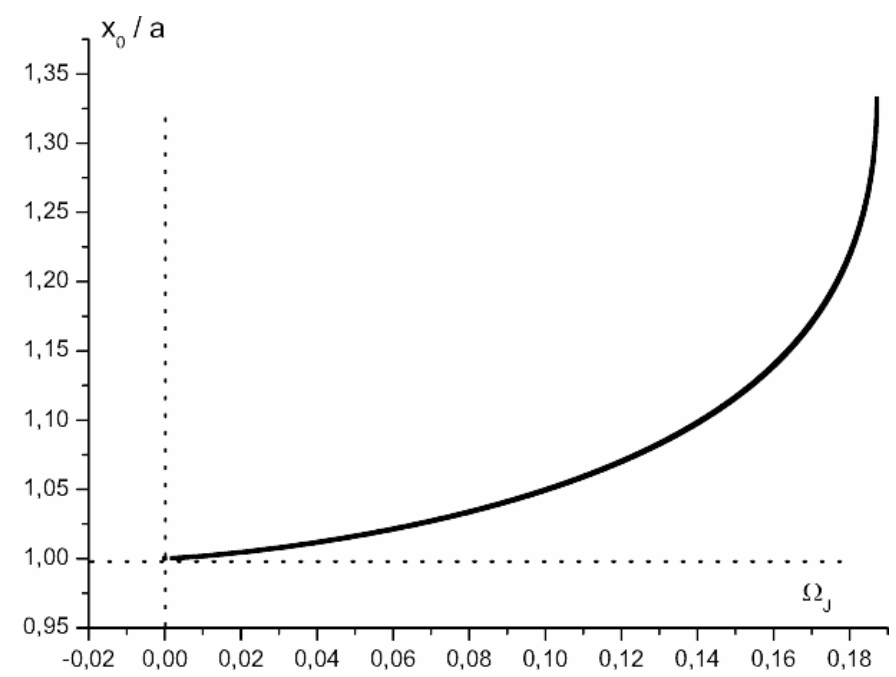

Fig. 3. The relative distance $x_{0} / a$ of the SEPs of a Jacobi ellipsoid from the center versus the parameter $\Omega_{\mathrm{J}}$.

a rubble-pile asteroid by a Jacobi ellipsoid is justified or not requires an independent study. The shape of real rubble-pile asteroids in general is far from the shape of a figure of equilibrium. Perhaps it could be considered as a first approximation to a rubble-pile asteroid (like the approximation of the shape of a monolithic asteroid by a triaxial ellipsoid). In Figs. 2 and 3 we give the dependence of the dimensionless distance $x_{0} / a$ of the SEPs of the Jacobi ellipsoid from its center on both the ratio $a / c$ of its semiaxes and the parameter $\Omega_{\mathrm{J}}$, where

$\Omega_{\mathrm{J}}=\frac{2 \pi}{k^{2} \rho T^{2}}$

(See, for example, Subbotin (1949) for a description of the parameters of Jacobi ellipsoids.) On the other hand, using Eq. (66), we have

$\frac{2 \pi}{k^{2} \rho T^{2}}=\Omega_{\mathrm{J}}=2 \frac{a}{c} \frac{b}{c} I_{1}$.

It is known that the value of the parameter $\Omega_{\mathrm{J}}$ does not exceed 0.1871 for the Jacobi ellipsoids and the ratio $a / c$ of the 


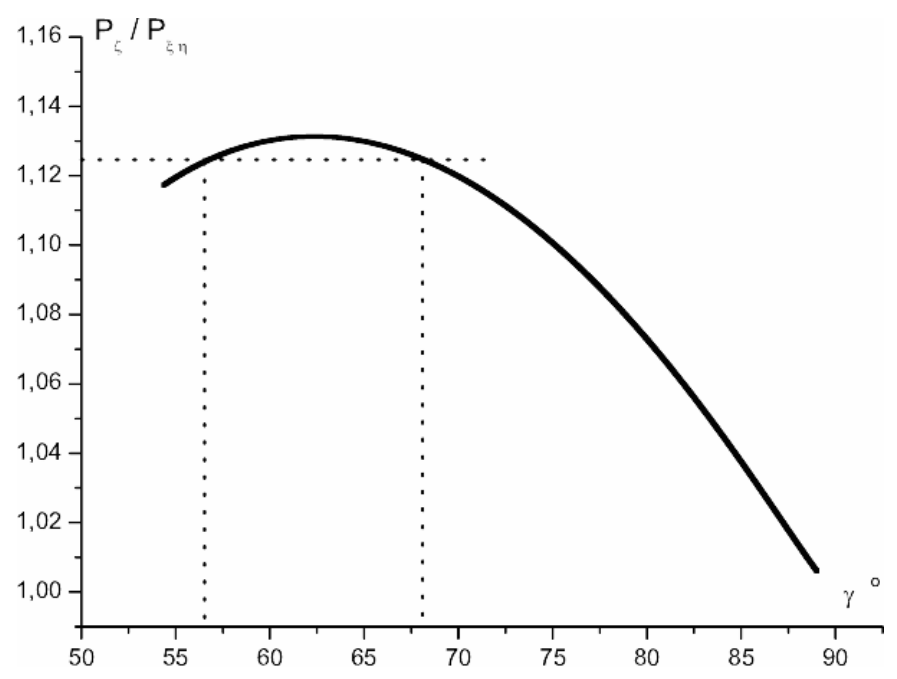

Fig. 4. The ratio of the periods $P_{\zeta}$ and $P_{\xi \eta}$ as a function of the parameter $\gamma$ of a Jacobi ellipsoid.

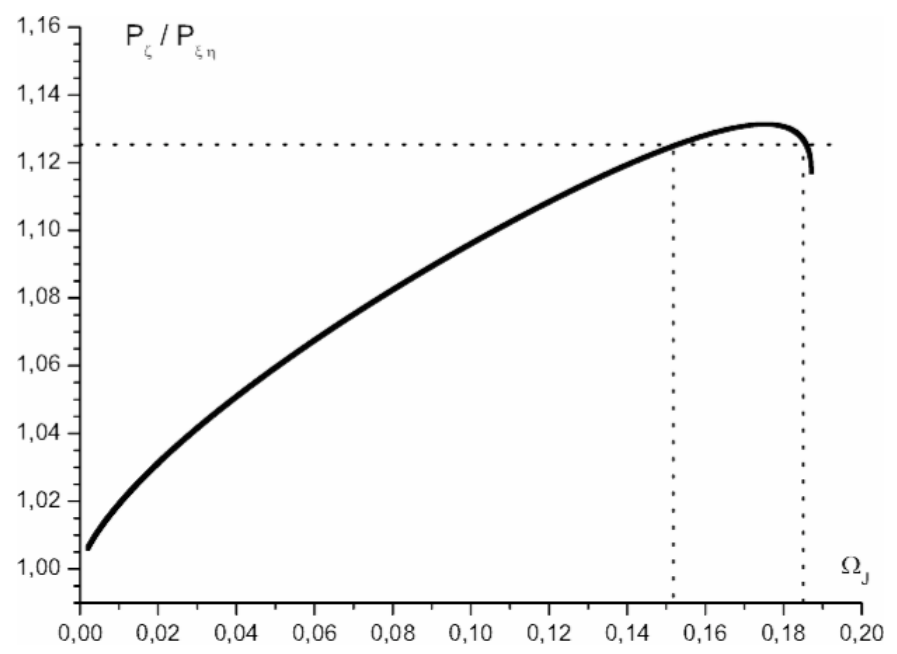

Fig. 5. The ratio of the periods $P_{\zeta}$ and $P_{\xi \eta}$ as a function of the parameter $\Omega_{\mathrm{J}}$ of a Jacobi ellipsoid.

Jacobi ellipsoid semiaxes $a>b>c$ varies from 1.716 to infinity (when the ellipsoid approaches a needle-like shape). In Figs. 2, 3 the SEP approaches the ellipsoid surface but does not touch it at any $\Omega_{\mathrm{J}}$ and for any ratio $a / c$ within the range admissible for a Jacobi ellipsoid.

In Figs. 4, 5 the ratio of periods $P_{\zeta}$ to $P_{\xi \eta}$ is plotted versus the parameters $\gamma$ and $\Omega_{\mathrm{J}}$ of the Jacobi ellipsoid, where $\gamma$ (varying from 54.3576 to 90 degrees) is computed from the equation

$c=a \cos \gamma$.

It can be noticed from Figs. 4, 5 that the only low-order commensurability for $m$ and $n$ smaller than 10 (Eq. (46)) at which a 3 -D periodic motion is possible (albeit unstable) is $m / n=9 / 8$ for $P_{\zeta} / P_{\xi \eta}=1.125$. It happens (see dotted lines in Figs. 4, 5) for two different values of the parameter $\gamma$ (or $\Omega_{\mathrm{J}}$ ) or for the following parameters of the Jacobi ellipsoid:

$\Omega_{\mathrm{J}}=0.1859, \gamma=56.9242, a: b: c=1.134: 1: 0.619 ;$

$\Omega_{\mathrm{J}}=0.1520, \gamma=67.9982, a: b: c=2.066: 1: 0.774$.
The relative distances of the SEPs from the center of the ellipsoid for these models are $x_{0} / a=1.394$ and $x_{0} / a=1.193$, respectively.

\section{Periodic motions in the vicinity of the CEPs}

Let us recall that at the CEPs of an asteroid approximated by a triaxial ellipsoid the following inequality is always fulfilled (see Eqs. (26), (27)):

$C=\widetilde{\Omega}_{x x} \widetilde{\Omega}_{y y}>0$,

therefore, the quantity $D$ in inequality (22) can be either positive or negative depending on the shape, density and spin rate of the asteroid. There are two roots $\widetilde{\sigma}_{1}^{2}, \widetilde{\sigma}_{2}^{2}$ of the quadratic Eq. (18):

$$
\begin{aligned}
& \widetilde{\sigma}_{1}^{2}=-\frac{B}{2}-\sqrt{\left(\frac{B}{2}\right)^{2}-C,} \\
& \widetilde{\sigma}_{2}^{2}=-\frac{B}{2}+\sqrt{\left(\frac{B}{2}\right)^{2}-C,}
\end{aligned}
$$

where $B$ is defined by Eq. (21). If at least one of the roots $\widetilde{\sigma}_{1}^{2}, \widetilde{\sigma}_{2}^{2}$ is negative, then (Eqs. (38), (39)) for a certain choice of the initial conditions, one family of planar periodic motions exists, because we have a pair of purely imaginary roots (Lyapunov 1950). Thus, the cases where the equatorial $(\xi \eta)$ projection of a 3-D motion is a closed periodic curve (namely, an ellipse (Eqs. (35), (36))) are the following:

(1) $D=0, B>0$;

(2) $D>0, B>0$.

First, let us consider case (1). We have $D=0$, that is, two equal roots

$\widetilde{\sigma}_{1}^{2}=\widetilde{\sigma}_{2}^{2}=-\frac{B}{2}$

From the theory of differential equations, a general solution of Eqs. (14), (15) of motion of a particle in the vicinity of the EP can then be expressed as

$\xi=\left(P_{1}+P_{3} t\right) \mathrm{e}^{i \omega \sqrt{\frac{B}{2}} t}+\left(P_{2}+P_{4} t\right) \mathrm{e}^{-i \omega \sqrt{\frac{B}{2}} t}$,

$\eta=\left(R_{1}+R_{3} t\right) \mathrm{e}^{i \omega \sqrt{\frac{B}{2}} t}+\left(R_{2}+R_{4} t\right) \mathrm{e}^{-i \omega \sqrt{\frac{B}{2}} t}$,

where $P_{i}, R_{i}$ are constants. Again, at a certain choice of the initial conditions (for example, when $P_{3}=P_{4}=R_{3}=R_{4}=0$ ) one family of planar periodic motions exists with the period of movement in the corresponding ellipse (which is the $\xi \eta$ projection of a potentially possible $3 \mathrm{DPM}$ ) equal to

$P_{1,2}=P=T \sqrt{\frac{2}{B}}$

Because (see condition (22)) the CEPs are unstable in the planar linear case at $D=0$, the corresponding 3DPM will also be unstable, i.e. even a small displacement will force a particle to spiral away from the CEP. In case (2) there are two families (of 


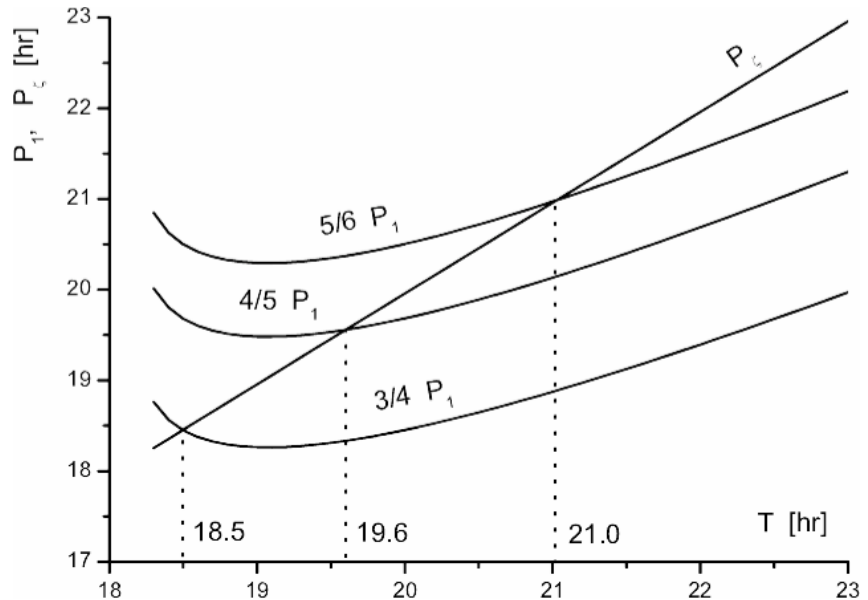

Fig. 6. Existence of low-order commensurabilities between the periods $P_{1}$ of the periodic movement in the ellipsoid equatorial plane and $P_{\zeta}$ of the periodic out-of-plane oscillation along the axis $\zeta$ in the vicinity of the CEP of an Ida-like TE as a function of the ellipsoid spin period $T$.

short and long period) of linearly stable elliptic periodic motions that are $\xi \eta$ projections of some 3-D periodic motions, because both roots $\widetilde{\sigma}_{1}^{2}, \widetilde{\sigma}_{2}^{2}$ are real and negative, and therefore we have two pairs of the purely imaginary roots $\widetilde{\sigma}_{1}, \widetilde{\sigma}_{2}, \widetilde{\sigma}_{3}, \widetilde{\sigma}_{4}$. The ratio of the semiaxes of the corresponding ellipses can be computed using Eq. (37), and the values of the short $\left(P_{1}\right)$ and the long $\left(P_{2}\right)$ periods of movement in the ellipses are (Eq. (36)):

$$
\begin{aligned}
& P_{1}=\frac{T}{\sqrt{\frac{B}{2}+\sqrt{\frac{B^{2}}{4}-C}}}, \\
& P_{2}=\frac{T}{\sqrt{\frac{B}{2}-\sqrt{\frac{B^{2}}{4}-C}}} .
\end{aligned}
$$

For a corresponding three-dimensional periodic motion in the vicinity of a CEP to exist it is necessary that (see Eq. (46))

$P_{1}=\frac{m_{1}}{n_{1}} P_{\zeta}, \quad P_{2}=\frac{m_{2}}{n_{2}} P_{\zeta}$,

or

$\frac{n_{1}}{m_{1}} P_{1}=P_{\zeta}, \quad \frac{n_{2}}{m_{2}} P_{2}=P_{\zeta}$,

where $m_{i}, n_{i}$ form an irreducible fraction and the period $P_{\zeta}$ is computed using Eq. (48). In Figs. 6, 7 the values of the spin period of an Ida-like asteroid are seen for which a 3DPM in the vicinity of the CEPs of an Ida-based ellipsoid is possible, that is, the equalities (87) hold. Let us recall that for the computations the model of a specific TE based on the asteroid 243 Ida: $a=28, b=12, c=10.5 \mathrm{~km}, \rho=2.5 \mathrm{~g} \mathrm{~cm}^{-3}$ (Beatty $1995)$ is used. The period of rotation of the TE was varied from $T=18.213 \mathrm{~h}$ (for which $D=0$ ) to approximately $60 \mathrm{~h}$. A 3DPM is possible for the spin period of the TE at which the curves $\frac{n_{1}}{m_{1}} P_{1}$ (Fig. 6) or $\frac{n_{2}}{m_{2}} P_{2}$ (Fig. 7) cross the curve $P_{\zeta}$. The intersections of these curves occur for the following low-order

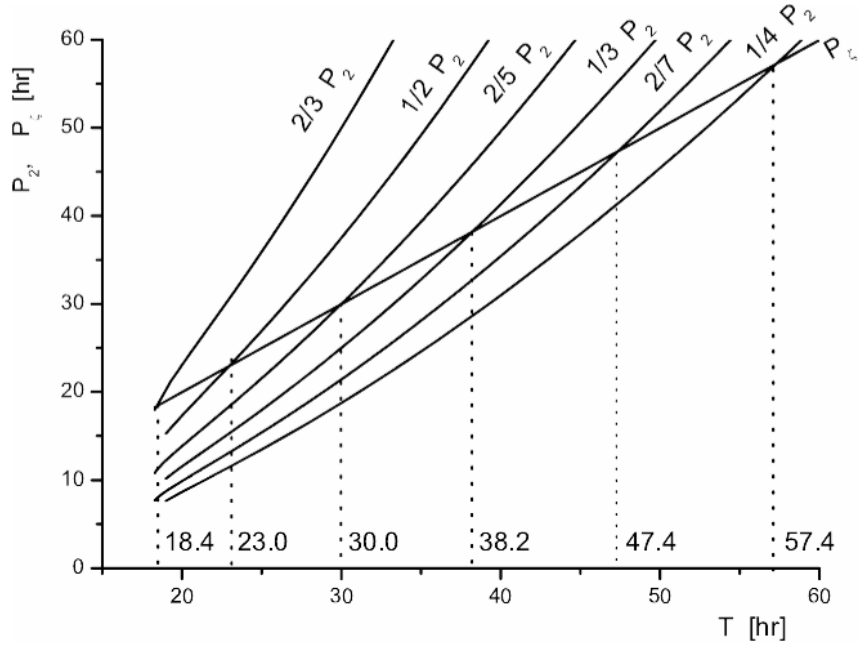

Fig. 7. Existence of low-order commensurabilities between the periods $P_{2}$ of the periodic movement in the ellipsoid equatorial plane and $P_{\zeta}$ of the periodic out-of-plane oscillation along the axis $\zeta$ in the vicinity of the CEP of an Ida-like TE as a function of the ellipsoid spin period $T$.

commensurabilities:

$\frac{P_{1}}{P_{\zeta}}=\frac{4}{3} ; \frac{5}{4} ; \frac{6}{5}$

$\frac{P_{2}}{P_{\zeta}}=\frac{3}{2} ; \frac{2}{1} ; \frac{5}{2} ; \frac{3}{1} ; \frac{7}{2}$.

For example, the 3DPMs at the commensurabilities (89) are potentially possible for the periods of rotation of the Ida-like asteroid approximately equal to $18.372,22.961,29.984,38.221,47.366$ h. In Figs. 8-12 the three-dimensional periodic orbits possible at these spin periods are presented as computed by numerical integration of the non-linear equations of motion in the $\xi \eta \zeta$ coordinate frame fixed in the CEP $\left(0, y_{0}, 0\right)$. The initial conditions are computed as the solutions of the corresponding variational equations (see Sect. 5). These motions are stable, as a consequence of the linear stability of the CEPs of a triaxial ellipsoid at $D>0, B>0$ : the displacement $\Delta$ (see Table 1 ) from the initial position in nearly 3 months does not exceed $8.3 \mathrm{~cm}$. The quantity $\Delta q$ in Table 1 represents the difference between the quantity $q$ predicted by the variational Eqs. (14)-(15) and computed by formula (37), and $q$ found as the axial ratio of the "real" ellipse computed by numerical integration of the non-linear equations of motion with the initial conditions (Eqs. (60)-(65)) obtained from the variational Eqs. (14)-(16). Let us recall that $q$ is the ratio of the semiaxes of the ellipse which is the $\xi \eta$ (equatorial) projection of a 3-D periodic motion in the vicinity of the EP. It is seen that this difference ranges between 0.0005 and 0.0018 only.

\section{Conclusion}

We describe a practical way of constructing the simplest symmetrical (with at least one plane of symmetry) 3-D periodic motions in the vicinity of the equilibrium points of a homogeneous rotating triaxial ellipsoid. The condition for a 3-D 


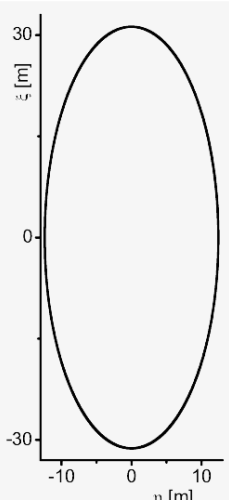

a)

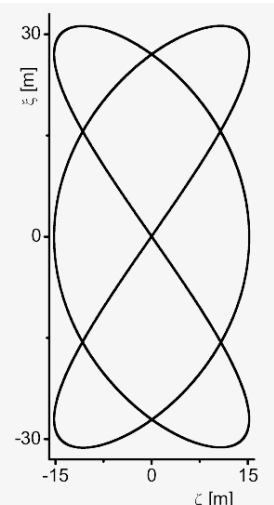

b)

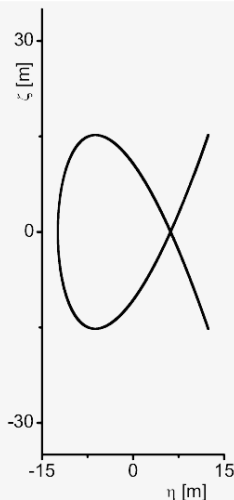

c)

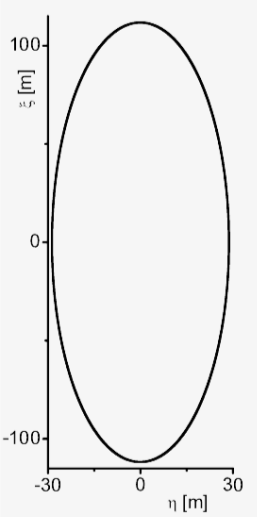

a)

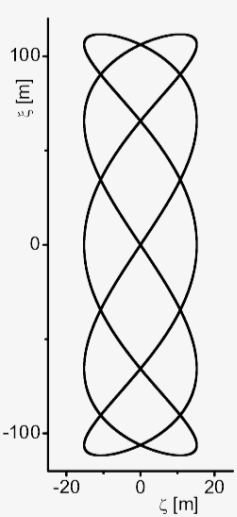

b)

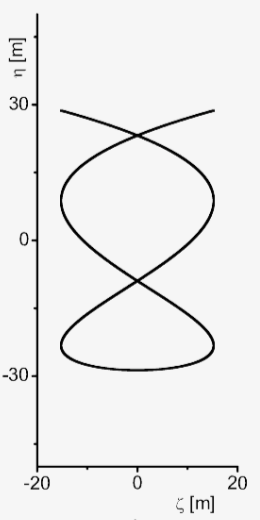

c) d)
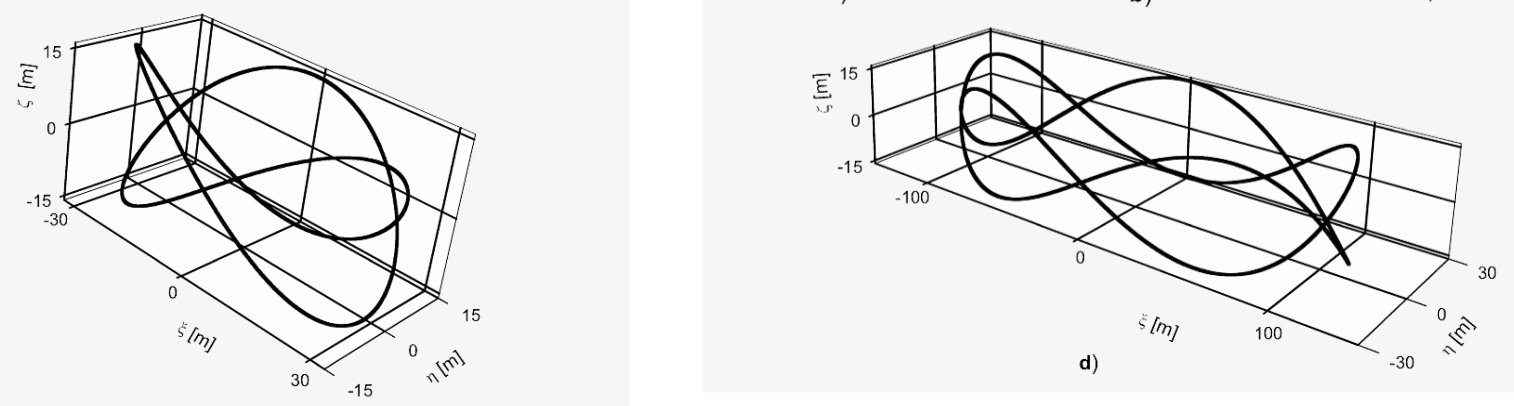

Fig. 10. A 3-D periodic motion in the reference frame fixed in the CEP of an Ida-like TE for the commensurability $P_{2} / P_{\zeta}=5 / 2$ and at an ellipsoid spin period of $T=29.977 \mathrm{~h}$. The period of the $3-\mathrm{D}$ motion is equal to $2 P_{2}=5 P_{\zeta}=149.697 \mathrm{~h}$. a) the $\xi \eta$ projection; b) the $\xi \zeta$ projection; c) the $\eta \zeta$ projection; d) the $3-\mathrm{D}$ representation.

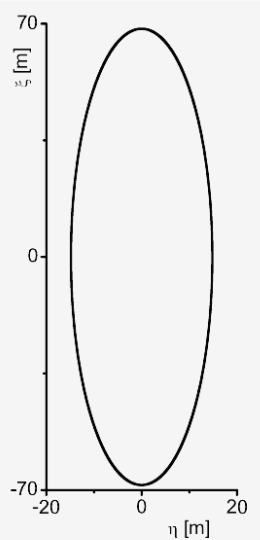

a)

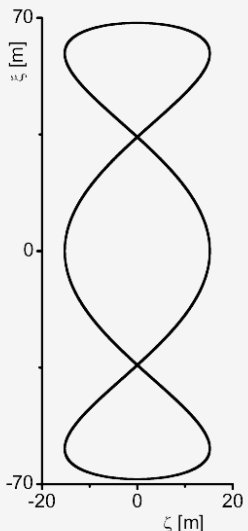

b)

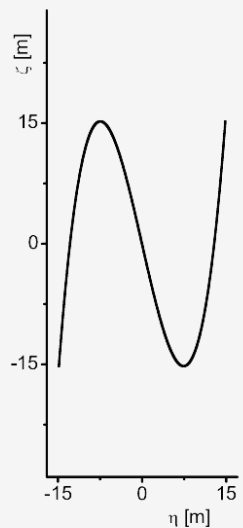

c)

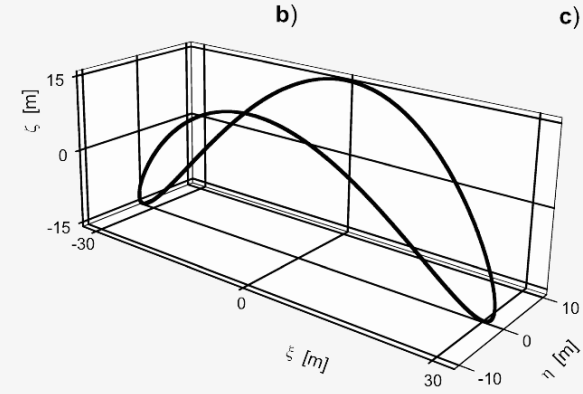

Fig. 9. A 3-D periodic motion in the reference frame fixed in the CEP of an Ida-like TE for the commensurability $P_{2} / P_{\zeta}=2 / 1$ and at an ellipsoid spin period of $T=22.955 \mathrm{~h}$. The period of the 3-D motion is equal to $P_{2}=2 P_{\zeta}=45.827 \mathrm{~h}$. a) the $\xi \eta$ projection; b) the $\xi \zeta$ projection; c) the $\eta \zeta$ projection; d) the 3-D representation.

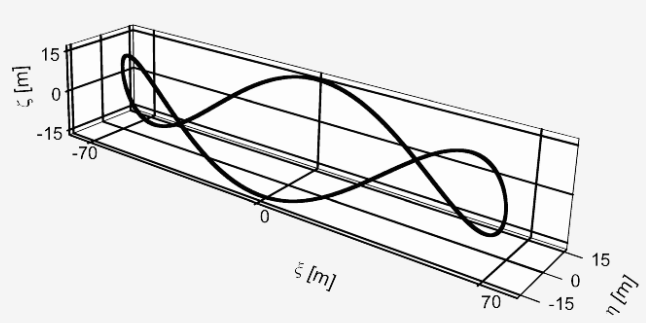

d)

Fig. 11. A 3-D periodic motion in the reference frame fixed in the CEP lipsoid spin period of $T=38.212 \mathrm{~h}$. The period of the $3-\mathrm{D}$ motion is equal to $P_{2}=3 P_{\zeta}=114.531 \mathrm{~h}$. a) the $\xi \eta$ projection; b) the $\xi \zeta$ projection; c) the $\eta \zeta$ projection; d) the $3-\mathrm{D}$ representation. of an Ida-like TE for the commensurability $P_{2} / P_{\zeta}=3 / 1$ and at an el- 


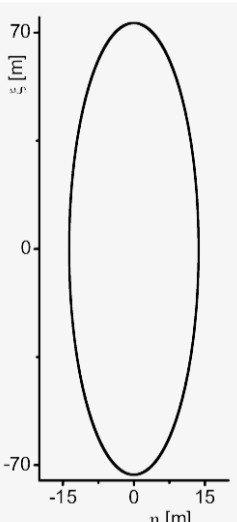

a)

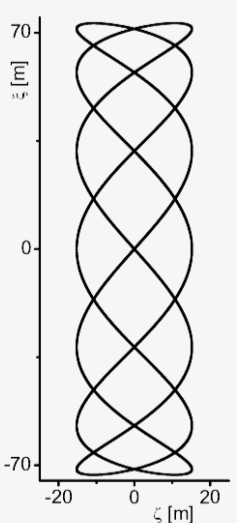

b)

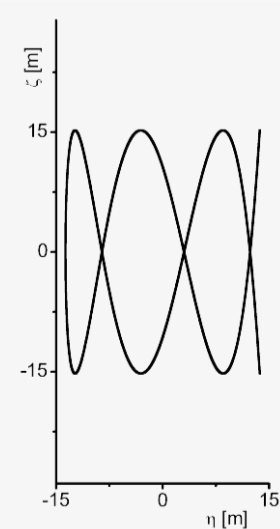

c)

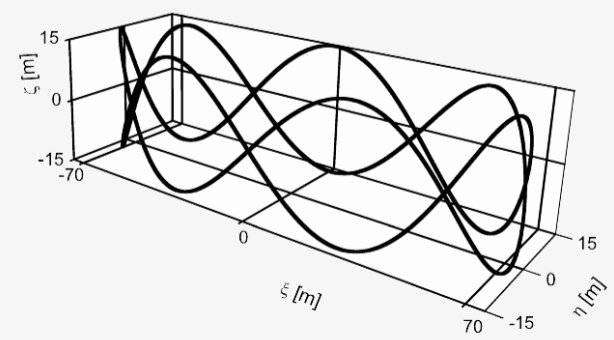

d)

Fig. 12. A 3-D periodic motion in the reference frame fixed in the CEP of an Ida-like TE for the commensurability $P_{2} / P_{\zeta}=7 / 2$ and at an ellipsoid spin period of $T=47.355 \mathrm{~h}$. The period of the 3-D motion is equal to $2 P_{2}=7 P_{\zeta}=114.531 \mathrm{~h}$. a) the $\xi \eta$ projection; b) the $\xi \zeta$ projection; c) the $\eta \zeta$ projection; d) the 3-D representation.

periodic solution to exist is pointed out depending on the spin period of the triaxial ellipsoid. All necessary formulae for computing the initial conditions and some parameters of a 3-D periodic motion using solutions of the variational equations of motion are given. It is shown that the $\xi \eta$ projection of a 3-D motion found in the way described is an ellipse. The formulae for computing the ratio $q$ of the semiaxes of the ellipse, the period of motion in it and the period of the 3-D periodic motion itself are derived. Some examples of 3-D periodic motions are computed by numerical integration of the non-linear equations of particle motion in the vicinity of the EPs of a specific TE based on the asteroid 243 Ida. The CEP-associated 3DPMs plotted in three orthographic projections together with their $3-\mathrm{D}$ representations and their parameters are listed in Table 1. The quantity $\Delta q$ is the deviation of the axial ratio of the "real" ellipse (the equatorial projection of a 3DPM obtained by numerical integration of non-linear equations of motion) from the axial ratio predicted by solving the variational equations. The quantity $\Delta$ in Table 1 is the deviation of the particle position from the initial position after a time interval of nearly 3 months, equal to an integer number of revolutions of the particle in the 3 -D periodic orbit. As the quantities $\Delta$ and $\Delta q$ range within $0.1-8.3 \mathrm{~cm}$ and $0.0023-0.0006$, the conclusion can be drawn that the initial conditions for 3-D motions obtained from the variational equations are a good approximation for computing the simplest 3DPMs by numerical integration of the non-linear equations.
Then we consider the general conditions for the period of a 3-D periodic motion (searched for in the current way) in the vicinity of the SEPs and CEPs to be commensurable or nearly commensurable with the ellipsoid (asteroid) spin period. It turned out, specifically, that for the motion in the vicinity of the CEPs such a commensurability occurs for asteroids having or approaching a two-axis ellipsoid shape. In the vicinity of the SEPs this happens, particularly, for nearly spherical asteroids.

Finally, a formula is derived which defines the spin period of the asteroid for which the SEPs are touching the asteroid surface and the destruction of the asteroid can start (if there are no internal cohesive forces in addition to gravity). It is shown that this period of rotation is the same for asteroids having the same density and the same axial ratios and is independent of their dimensions and masses. Some comments are made concerning the conditions for a 3-D periodic motion to exist in the vicinity of the SEPs of a Jacobi ellipsoid (which could represent rubble-pile asteroids or asteroid planetesimals).

Acknowledgements. I would like to thank Drs. Yu. V. Batrakov and V. K. Abalakin for their interest in this work and useful comments and the referee Dr. G. Contopoulos for helpful criticism and valuable corrections.

\section{References}

Abalakin, V. K. 1957, Bull. ITA, 6, 8, 543 (in Russian)

Aksenov, E. P. 1960, Soobscheniya Shternberg Gos. Astron. Instit., 115, 44 (in Russian)

Antonov, V. A. 1961, Vestnik Leningradskogo Universiteta, 13, 3, 157 (in Russian)

Batrakov, Yu. V. 1957, Bull. ITA, 6, 8, 524 (in Russian)

Beatty, J. K. 1995, Sky, \& Telescope, January, 20

Chauvineau, B., Farinella, P., \& Mignard, F. 1993, Icarus, 105, 2, 370

Colin, J. 1979, A\&A, 76, 3, 356

Contopoulos, G. 1973, ApJ, 181, 3, 657

Danby, J. M. A. 1965, AJ, 70, 7, 501

Duboshin, G. N. 1961, Theory of Gravitational Attraction (Moscow), 288, (in Russian)

Kosenko, I. I. 1981, Cosmic Research, 19, 2, 200 (in Russian)

Kosenko, I. I. 1981, Applied Math. and Mech., 45, 1, 26 (in Russian) Lyapunov, A. M. 1950, General Problem of Stability of Motion (GITTL, Moscow - Leningrad)

Lynden-Bell, D. 1979, MNRAS, 187, 1, 101

Nikolaev, S. I. 1968, Ukrain. Math. J., 20, 5, 661 (in Russian)

Nikolaev, S. I. 1969, Math. Physics, 6, 134 (in Russian)

Nikolaev, S. I. 1972, Bull. ITA, 13, 4, 215 (in Russian)

Papayannopoulos, Th. 1979, A\&A, 77, 75

Papayannopoulos, Th. 1979, A\&A, 79, 197

Pfenniger, D. 1990, A\&A, 230, 55

Scheeres, D. J. 1993, Advances in Non-Linear Astrodynamics (Univ. of Minnesota), Nov., 8

Scheeres, D. J. 1994, Icarus, 110, 2, 225

Schwarz, M. P. 1984, MNRAS, 209, 1, 93

Subbotin, M. F. 1937, Manual on Celestial Mechanics, II (Moscow) (in Russian)

Subbotin, M. F. 1949, Manual on Celestial Mechanics, III (Moscow) (in Russian)

Vasilkova, O. 1999, Proc. Inst. Applied Astron. of RAS, 4, 246 (in Russian)

Zhuravlev, S. G. 1972, Cel. Mech., 6, 3, 255

Zhuravlev, S. G. 1973, Cel. Mech., 8, 1, 75

Zhuravlev, S. G. 1974, (Russian) Astron. zhurnal, 51, 6, 1330 (in Russian) 